\title{
Medial sural artery perforator flap: a challenging free flap
}

\author{
Navid Mohamadpour Toyserkani ${ }^{1}$ • Jens Ahm Sørensen ${ }^{1}$
}

Received: 9 April 2015 / Accepted: 12 May 2015 / Published online: 24 May 2015

(C) Springer-Verlag Berlin Heidelberg 2015

\begin{abstract}
Background Oral and extremity defect reconstruction can often require a flap that is thin, and traditionally, the radial forearm free flap has been used, however, this has significant donor site morbidity. Over the last decade, the medial sural artery perforator (MSAP) flap has emerged as a possible alternative with lower donor site morbidity. We present our experiences and review the literature regarding this promising but challenging flap.

Methods The study was a retrospective case series in a university hospital setting. All patients who had a MSAP flap performed at our institution were included until March 2015, and their data was retrieved from electronic patient records. Results In total, ten patients were reconstructed with a MSAP flap for floor of mouth (eight) and lower extremity (two) defect reconstruction. The median flap dimensions were as follows: $10 \mathrm{~cm}$ (range 7-14 cm), width $5 \mathrm{~cm}$ (range $3.5-8 \mathrm{~cm}$ ), thickness $5 \mathrm{~mm}$ (range 4-8 $\mathrm{mm}$ ), and pedicle length $10 \mathrm{~cm}$ (range 8-12 cm). In one case, the procedure was abandoned because of very small perforators and another flap was used. In two cases, late onset of venous congestion occurred which could not be salvaged. There were no donor site complaints. Conclusions The MSAP flap is an ideal flap when a thin free flap is needed with lower donor site morbidity than alternative solutions. There seems to be a higher rate of late onset of venous thrombosis compared with more established flaps. Therefore, this flap should be monitored more closely for
\end{abstract}

Jens Ahm Sørensen

jens.sorensen@rsyd.dk

1 Department of Plastic and Reconstructive Surgery, Odense University Hospital, Sdr. Boulevard 29, DK-5000 Odense C, Denmark venous problems and we recommend performing two venous anastomoses when using this flap.

Level of Evidence: Level IV, therapeutic study.

Keywords Medial sural artery perforator flap · Microsurgery · Complication $\cdot$ Floor of mouth reconstruction $\cdot$ Distal limb reconstruction

\section{Introduction}

Reconstruction with free flaps is a necessity when defects become too large for more simple reconstruction options such as skin grafting and local flap options. Many different free flaps have been described throughout the years each with their own strengths and weaknesses.

Several free flap options are available for oral reconstruction (without mandible defects), and the dominant choices today are the anterolateral thigh (ALT) flap and the radial free forearm flap (RFFF). Since the anterolateral thigh flap was first reported by Song et al. in 1984 [1], its popularity has steadily increased and is now one of the most used flaps for head and neck reconstructions. This is largely due to its reliability, versatility, long vascular pedicle with optimal diameter for anastomosis, and the possibility for harvesting a large skin territory. Furthermore, the donor site morbidity is very low [2]. The downside of this flap is its bulkiness which for some defects can be too much. The flap can be thinned but still with limits, and often, it is most appropriate to wait for a secondary debulking procedure $[3,4]$. On the other hand, the radial forearm flap is also very reliable but has much larger donor site morbidity and the defect in many cases requires split skin grafting [5]. However, the flap is very thin which in some reconstructions is a necessity. What has been missing in the armamentarium of a microsurgeon is a flap that combines the 
thinness of the RFFF with the low donor site morbidity of the ALT flap, and the medial sural perforator flap has emerged as a possible candidate to fill this gap.

The medial sural perforator (MSAP) flap was first described in 2001 by Cavadas et al. [6] and has since been described for both extremity and head and neck reconstructions. At our institution, the ALT flap is the flap of choice for head and neck reconstructions, but since 2010, we have used the MSAP flap for oral reconstruction of floor of mouth defects where the ALT flap was believed to be too bulky. In general, we try to avoid the RFFF because of the high donor site morbidity. We will describe our case series and compare and discuss our experiences with published results so far.

\section{Methods}

This study was conducted as a retrospective case series in a university hospital setting. All patients between December 2010 and March 2015 with oral cancer reconstructed with MSAP flap were included. The study was retrospective and did therefore not require approval from the regional ethics committee.

The medical charts were reviewed to record the following data: age, sex, etiology, defect area, flap option, flap size, recipient vessels, donor site closure, and postoperative morbidity.

For a two-team approach, the patient was usually placed in a supine position with the hip abducted and knee flexed in a frog-like position. A hand-held Doppler probe was used for mapping out the perforators from the medial gastrocnemius. The main sizable perforator was located $8 \mathrm{~cm}$ distal to the popliteal crease located on a line from the middle of the popliteal crease to the medial calcaneus. If more than one perforator was present, they were usually located distally to this up $15 \mathrm{~cm}$ distal to the popliteal crease. The medial border of the flap is elevated to confirm the location and size of the perforators. When one or two sizable perforators are identified, the opposite border is then incised and the flap elevated. The pedicle is then freed from the medial head of the gastrocnemius muscle through an intramuscular dissection. Hemostasis was meticulously carried out using bipolar cautery. Basically, the course of the main trunk of the vascular pedicle is parallel to the gastrocnemius muscle fibers and can be exposed by splitting them. The pedicle may be dissected 9 to $16 \mathrm{~cm}$ in length (see Fig. 1 for an intraoperative photo). When we started using this particular flap, the superficial vein was not routinely harvested but we have changed the procedure, and if possible, we harvest a superficial vein to secure venous outflow (see Fig. 2 for an intraoperative photo where the subcutaneous vein is harvested). We have also added flow coupler as a standard monitoring system for these flaps in addition to microdialysis and clinical evaluation.

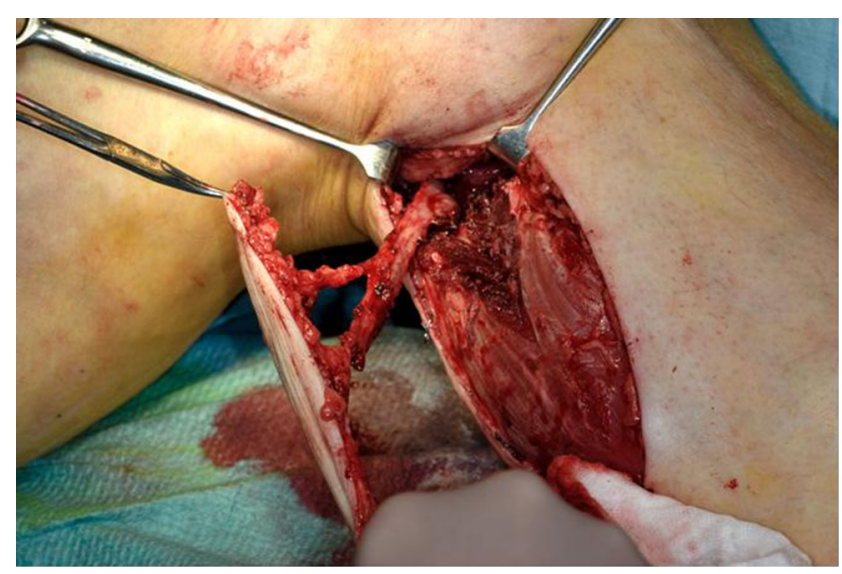

Fig. 1 The MSAP flap after intramuscular dissection with two perforators

Donor site was closed primarily which was possible in all our cases except one which was the largest with a width of $8 \mathrm{~cm}$ in which case a split skin graft was used (see Fig. 3 for a typical result at the donor site).

\section{Results}

From December 2010 until March 2015, eight microvascular MSAP free flaps were performed for intraoral defects after removal of floor of mouth cancer. In two cases, we used the MSAP flap for plantar reconstruction. Nine were males and one was female. The median age at time of surgery was 52 years (range $45-66$ years).

The median flap length was $10 \mathrm{~cm}$ (range 7-14 cm), and median flap width was $5 \mathrm{~cm}$ (range $3.5-8 \mathrm{~cm}$ ). The median artery diameter was $1.25 \mathrm{~mm}$ (range $1-1.5 \mathrm{~mm}$ ), and the median vein diameter was $2 \mathrm{~mm}$ (range 1.5-2.5). The median pedicle length was $10 \mathrm{~cm}$ (range $8-12 \mathrm{~cm}$ ). The median thickness was $5 \mathrm{~mm}$ (range 4-8 $\mathrm{mm}$ ). Either the facial or superior thyroid vessels were used for anastomosis for oral

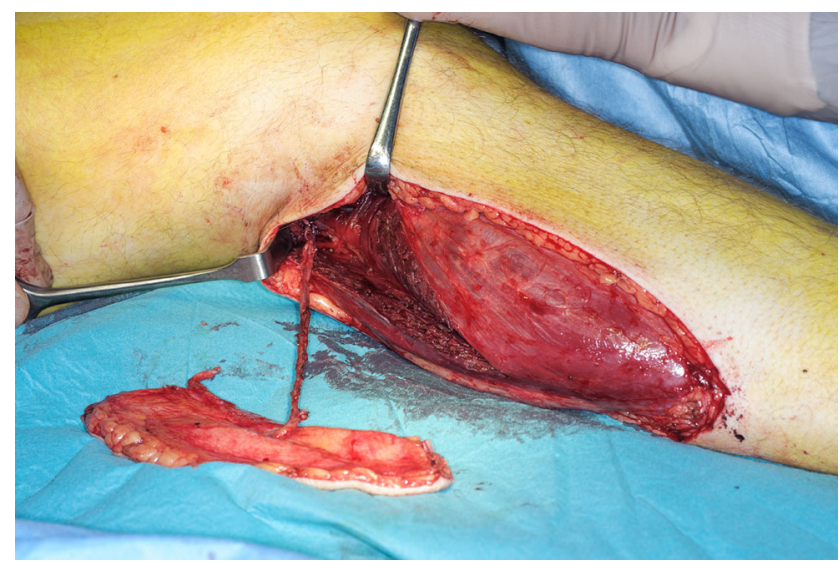

Fig. 2 The MSAP flap after dissection including the harvested subcutaneous vein 


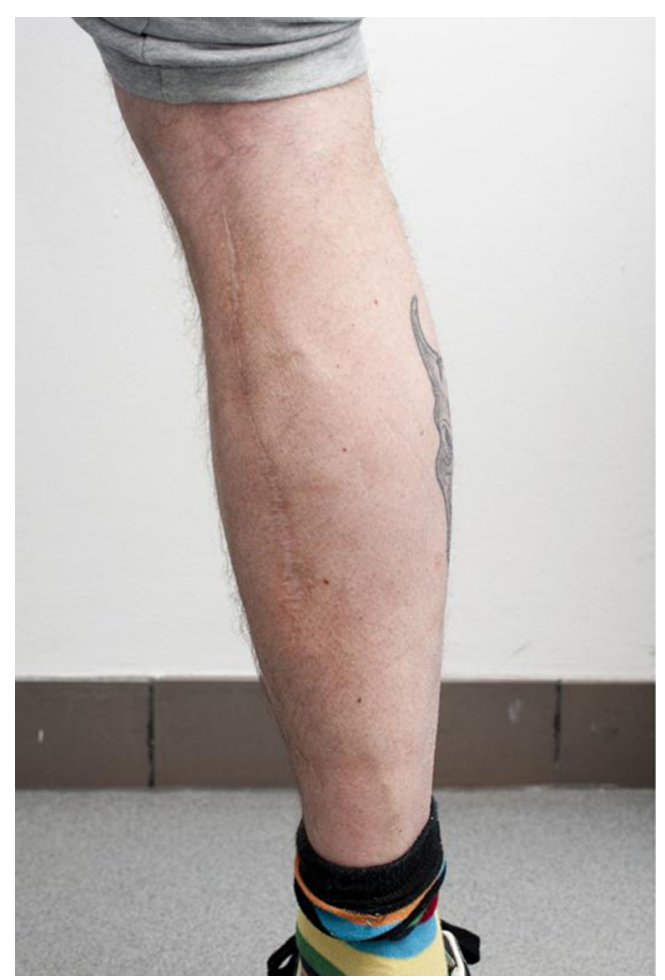

Fig. 3 Typical postoperative scar at donor site 3 months after surgery

reconstruction. For the plantar defects, the posterior tibial vessels were used.

We had six uncomplicated cases, one case with venous problems that was salvaged, two failures, and one case was abandoned intraoperatively while elevating the flap as the perforators were extremely small and converted to an ALT flap.

Our first failure was an $8 \times 4-\mathrm{cm}$ MSAP flap raised on two perforators and a $10-\mathrm{cm}$ pedicle. The operation was uncomplicated, and there were fine microdialysis values immediately postoperatively. Approximately $48 \mathrm{~h}$ postoperatively, the flap became cyanotic and the patient was taken to the operating room where the vein was found thrombosed. The flap could not be salvaged, and an ALT flap was made instead.

The second failure was a $6 \times 10$-cm MSAP flap with an 8 $\mathrm{cm}$ pedicle. The flap was raised on a single relatively thin perforator, however, the flap was well perfused during surgery even after $72 \mathrm{~min}$ of warm ischemia time. Approximately $36 \mathrm{~h}$ postoperatively, the microdialysis values begin to deteriorate indicating a venous anastomosis problem and the flap gradually became darker. The patient was taken to the operating room acutely where no stasis of the pedicle was found. The vein anastomosis was found torqued which was loosened, but afterwards, no obvious flow was evident. No venous thrombosis was found, and by the end, it seemed as the flow was gradually improving. Few days later, the flap had failed and an ALT flap was performed.

The flap that was salvaged was a $14 \times 8$-cm MSAP flap raised on a single perforator with a $12-\mathrm{cm}$ pedicle. The flap was supercharged with a subcutaneous vein. The microdialysis and flow were fine at surgery, however, about $8 \mathrm{~h}$ postoperatively, the flow signal stopped and thereafter the microdialysis values deteriorated. The flap was reoperated acutely, and both venous anastomoses were found to be with significantly diminished flow possibly because of the too tight closure around the recipient site. The anastomoses were redone, the site was closed under less tension, and the flap survived fully with no further complications.

No donor site complaints were noted from the patients at 3month follow up regarding the scar or any functional problems.

\section{Discussion}

The success of a reconstruction is dependent on the size and location of the defect, the flap option, and correct threedimensional application of the flap. Especially, reconstruction of oral defects is a demanding task which aims to restore the functions of the mouth such as articulation, mastication, and airway protection. The most widely used flaps for oral reconstruction are the ALT and RFFF flaps.

The ALT flap has the downside of sometimes being too bulky, and the radial forearm flap has great donor site morbidity, but the flap itself is of great quality and size. The MSAP flap offers the potential best of both worlds as the donor site morbidity is quite low as with the ALT flap, but the thickness is rather comparable to the RF flap.

Kao et al. compared the results of the radial free forearm flap (RFFF) with the MSAP, and they found $100 \%$ survival of both groups [7]. As could have been expected, the subjective outcome regarding donor site morbidity was superior for the MSAP flap. Kao et al. later described a case series of 26 MSAP flaps (extension of their previous publication) for head and neck reconstruction of which one flap failed because of venous insufficiency 2 days postoperatively [8].

Choi et al. described a case series of 20 patients with MSAP flap for oral cancer reconstruction of which two flaps failed, and an alternative flap had to be used [9]. The first case of flap failure was raised on a single very small diameter perforator which was confirmed to be pulsating although it was noted that the route of the pedicle had a very rightangled shape near the medial popliteal artery. Twenty-four hours after surgery, the flap turned purple and the flap could not be salvaged. The second case was raised on two arteries and three veins, but about $48 \mathrm{~h}$ after surgery, the flap turned purple but the perforator area of the pedicle was clotted completely and could not be salvaged. Chen et al. described their series of 12 patients with MSAP flap for oral cancer reconstruction of which one flap failed [10]. This failure happened on the fifth postoperative day and was due to venous insufficiency, but no salvage was possible. 
Chen et al. described their series of MSAP flaps for distal limb defects of 11 patients of which one flap failed [11]. The failed flap developed venous congestion and could not be salvaged. The underlying reason was suspected of being kinking of the perforator or that the diameter of the perforator was too small for venous drainage. Wang et al. presented their case series of MSAP flaps for distal limb reconstruction including 34 flaps of which five underwent partial necrosis [12]. From their description of the partial failure cases, a venous problem arose (partial purpling with bubbles and subcutaneous hematocele), which was handled conservatively with regular wound management. Kim et al. described their series of patients with plantar defects reconstructed with the MSAP flap [13]. Eleven patients were included and all flaps survived, however, one flap developed venous congestion but was managed with leech therapy. Hallock has performed 14 MSAP flaps for ipsilateral lower extremity defects of which one failed because of venous congestion which was salvaged with another free flap [14].

Only two case series did not have any venous failures, but instead, each series had one arterial flap failure. He et al. presented their case series of nine patients where they had used the MSAP flap for oral reconstruction using preoperative computed tomography angiography (CTA) planning [15]. They found a good correlation between the locations of the perforators when comparing the CTA with intraoperative findings. One case failed because of arterial insufficiency $60 \mathrm{~h}$ postoperatively which could not be salvaged.

The MSAP flap has also garnered attention for both upper and lower extremity reconstructions. Lin et al. have described a series of 14 patients where the MSAP flap was used for hand reconstruction [16]. Only one flap failed, which was attributed to a large difference in donor and recipient artery diameter (1:4) as well as vasospasm. No cases with venous problems were described. See Table 1 for an overview of all case series described, where the MSAP flap has been used and failure rates have been mentioned.

Many of the cases of failure described in the literature are of somewhat late onset and similar to our own experiences, and one has to wonder what the underlying mechanism is as these failures are extremely rare when using the more established free flaps. Seven out of nine attempts of the use of this flap were successful which is markedly lower than any other flaps used at this institution. The usual failure rate of more established free flaps such as the ALT flap or RFFF is reported to be much lower in the literature in the range of about $2-4 \%[17,18]$. There is an obvious risk of sample bias with such a low number of cases, however when all reported MSAP flaps outcomes are pooled, we still see a failure rate of $9.9 \%$ which is higher than normally reported figures for more established flaps. A venous problem was the cause of failure in $87.5 \%$ of all described failures.

Using a new flap to reconstruct a specific defect will always be associated with a learning curve. We had no problems during the flap elevation, and the late onset of flap failure raises the suspicion that something in the flap itself may in some cases be insufficient. We found that the perforator quality was much more variable compared with the ALT flap. The majority of reported failures have been late onset venous thrombosis. This could indicate that the accompanying venae comitantes in some cases may be inadequate of draining the flap if only one venous anastomosis is performed. A potential solution could be to supercharge the flap to secure venous outflow, which has been described previously by Hallock et al. [19]. The primary venous drainage system could be identified preoperatively by clamping the veins temporarily and observing the flap clinically to determine which system is the main venous outlet. Since our review of the literature and our two failures, we always strive to perform two venous anastomoses for the MSAP flap preferably using a
Table 1 List of all published case series and their failure rates

\begin{tabular}{lllll}
\hline & Total cases & Partial failures & Total failures & Failure rate \\
\hline Wang et al. [14] & 34 & 5 (venous $^{\mathrm{a}}$ ) & 0 & $14.7 \%$ \\
Chen et al. [10] & 12 & 0 & 1 (venous) & $8.3 \%$ \\
Choi et al. [9] & 20 & 0 & 2 (venous $\left.{ }^{\mathrm{a}}\right)$ & $10.0 \%$ \\
Lin et al. [12] & 14 & 0 & 1 (arterial) & $7.1 \%$ \\
Kim et al. [15] & 11 & 1 (venous) & 0 & $9.1 \%$ \\
Chen et al. [13] & 11 & 0 & 1 (venous) & $9.1 \%$ \\
Kao et al. [8] & 26 & 0 & 1 (venous) & $3.4 \%$ \\
Hallock [16] & 14 & 0 & 1 (venous) & $7.1 \%$ \\
He et al. [11] & 9 & 0 & 1 (arterial) & $11.1 \%$ \\
Our own & 9 & 0 & 2 (venous) & $22.2 \%$ \\
Total & 162 & 6 & 10 & $9.9 \%$ \\
\hline
\end{tabular}

The failure rates include all partial and total failures described

${ }^{a}$ Interpretation of underlying cause was based on symptoms described in their case series as no outright cause was mentioned 
subcutaneous vein if possible or using both venae comitantes. This is in line with previous reports that performing two anastomoses on two separate venous systems results in less venous insufficiency problems [20]. Additionally, we have added a new monitoring system, flow coupler, on what we estimate to be the main venous anastomosis. This has been added on top of already routine flap monitoring with clinical evaluation and microdialysis, which is very easy to analyze and can be used even by less experienced nursing staff for monitoring of flaps, that can be difficult to monitor clinically [21]. The flow coupler was first introduced in 2010 and has been reported to have a false-positive rate of about $5-10 \%[22,23]$.

The advantages of the medial sural perforator flap include the possibility of a two-team approach as the flap can be harvested with the patient in the supine position and the hip abducted and knee flexed. The flap donor site can be closed directly if the width is not too large (our limit has been $6 \mathrm{~cm}$ in width for direct closure). The pedicle is of sufficient length, and the vessels mostly have a suitable diameter. No major vessels are sacrificed. The donor site morbidity is minimal when compared to the obvious alternative RFFF, and reported donor site complaints include hypertrophic scarring, itching, and transitory functional impairment [7]. The downfall is a tedious surgical dissection intramuscularly and a possible diminished venous outflow.

In conclusion, we have presented our results with the medial sural perforator flap. This flap has similar characteristics to the RFFF but with lower donor site morbidity and more tedious elevation process. We use it in situations where the ALT flap is too bulky. The risk of flap failure due to late onset venous thrombosis seems to be somewhat higher than the more established free flaps. Therefor, this flap should be monitored more closely for venous problems and we recommend performing two venous anastomoses when using this flap. Given the low morbidity of the flap and the ideal characteristic of the tissue, this could very well become the flap of choice for floor of mouth and tongue reconstructions. Patients should be informed about the higher risk of failure when using this flap.

Ethical standards This study was performed as a retrospective case series, and therefore, approval from the regional ethical committee was not necessary.

Conflict of interest Navid Mohamadpour Toyserkani and Jens Ahm Sørensen declare that they have no conflict of interest.

Patient consent Informed consent has been obtained prior to the use of the photographs.

\section{References}

1. Song YG, Chen GZ, Song YL (1984) The free thigh flap: a new free flap concept based on the septocutaneous artery. Br J Plast Surg 37: 149-59
2. Collins J, Ayeni O, Thoma A (2012) A systematic review of anterolateral thigh flap donor site morbidity. Can J Plast Surg 20:1723

3. Agostini T, Lazzeri D, Spinelli G (2014) Anterolateral thigh flap thinning: techniques and complications. Ann Plast Surg 72:246-52

4. Sharabi SE, Hatef DA, Koshy JC, Jain A, Cole PD, Hollier LH (2010) Is primary thinning of the anterolateral thigh flap recommended? Ann Plast Surg 65:555-9

5. Richardson D, Fisher SE, Vaughan ED, Brown JS (1997) Radial forearm flap donor-site complications and morbidity: a prospective study. Plast Reconstr Surg 99:109-15

6. Cavadas PC, Sanz-Giménez-Rico JR, Gutierrez-de la Cámara A, Navarro-Monzonís A, Soler-Nomdedeu S, Martínez-Soriano F (2001) The medial sural artery perforator free flap. Plast Reconstr Surg 108:1609-15, discussion 1616-7

7. Kao H-K, Chang K-P, Wei F-C, Cheng M-H (2009) Comparison of the medial sural artery perforator flap with the radial forearm flap for head and neck reconstructions. Plast Reconstr Surg 124:112532

8. Kao H-K, Chang K-P, Chen Y-A, Wei F-C, Cheng M-H (2010) Anatomical basis and versatile application of the free medial sural artery perforator flap for head and neck reconstruction. Plast Reconstr Surg 125:1135-45

9. Choi JW, Nam SY, Choi SH, Roh JL, Kim SY, Hong JP (2013) Applications of medial sural perforator free flap for head and neck reconstructions. J Reconstr Microsurg 29:437-42

10. Chen S-L, Yu C-C, Chang M-C, Deng S-C, Wu Y-S, Chen T (2008) Medial sural artery perforator flap for intraoral reconstruction following cancer ablation. Ann Plast Surg 61:274-9

11. Chen S-L, Chen T, Lee C (2005) Free medial sural artery perforator flap for resurfacing distal limb defects. J Trauma 58:323-7

12. Wang X, Mei J, Pan J, Chen H, Zhang W, Tang M (2013) Reconstruction of distal limb defects with the free medial sural artery perforator flap. Plast Reconstr Surg 131:95-105

13. Kim ES, Hwang JH, Kim KS, Lee SY (2009) Plantar reconstruction using the medial sural artery perforator free flap. Ann Plast Surg 62: 679-84

14. Hallock GG (2014) Medial sural artery perforator free flap: legitimate use as a solution for the ipsilateral distal lower extremity defect. J Reconstr Microsurg 30:187-92

15. He Y, Jin S-F, Zhang Z-Y, Feng S-Q, Zhang C-P, Zhang Y-X (2014) A prospective study of medial sural artery perforator flap with computed tomographic angiography-aided design in tongue reconstruction. J Oral Maxillofac Surg 72:2351-65

16. Lin C-H, Lin C-H, Lin Y-T, Hsu C-C, Ng TW, Wei F-C (2011) The medial sural artery perforator flap: a versatile donor site for hand reconstruction. J Trauma 70:736-43

17. Frederick JW, Sweeny L, Carroll WR, Peters GE, Rosenthal EL (2013) Outcomes in head and neck reconstruction by surgical site and donor site. Laryngoscope 123:1612-7

18. Celik N, Wei F-C, Lin C-H, Cheng M-H, Chen H-C, Jeng S-F et al (2002) Technique and strategy in anterolateral thigh perforator flap surgery, based on an analysis of 15 complete and partial failures in 439 cases. Plast Reconstr Surg 109:2211-6, discussion 2217-8

19. Hallock GG, Sano K (2004) The medial sural medial gastrocnemius perforator free flap: an "ideal" prone position skin flap. Ann Plast Surg 52:184-7

20. Ichinose A, Terashi H, Nakahara M, Sugimoto I, Hashikawa K, Nomura T et al (2004) Do multiple venous anastomoses reduce risk of thrombosis in free-flap transfer? Efficacy of dual anastomoses of separate venous systems. Ann Plast Surg 52:61-3

21. Setälä L, Papp A, Romppanen E-L, Mustonen P, Berg L, Härmä M (2006) Microdialysis detects postoperative perfusion failure in microvascular flaps. J Reconstr Microsurg 22:87-96 
22. Kempton SJ, Poore SO, Chen JT, Afifi AM (2014) Free flap monitoring using an implantable anastomotic venous flow coupler: analysis of 119 consecutive abdominal-based free flaps for breast reconstruction. Microsurg. doi:10.1002/micr.22341
23. Zhang T, Dyalram-Silverberg D, Bui T, Caccamese JF, Lubek JE (2012) Analysis of an implantable venous anastomotic flow coupler: experience in head and neck free flap reconstruction. Int J Oral Maxillofac Surg Int Assoc Oral Maxillofac Surg 41:751-5 